\title{
A Relationship of Interstitial Cystitis and Atopy
}

\section{Steven Smith MD/MS FAAAA * and Holly Hoffer Reed BSN, RN}

Accrediated Allergy and Food Intolerence Center, Louisville, Kentucky

\begin{abstract}
Interstitial cystitis is a painful bladder condition, which has been recognized for over 100 years but has still been classified as idiopathic. In the search for causes and treatments, atopy (allergy) has been suggested. No large clinical observations have been reported to date. We have evaluated a large group of interstitial cystitis patients for allergic contributions and present the results in this clinical communication for the consideration of the reader.
\end{abstract}

Keywords: Atopy; Omalizumab; Th2 Cytokine inhibitor; Antihistamine; Leukotriene modifiers; Interstitial cystitis; Skin prick test; Elimination challenge; Diet; Food allergy; Pelvic floor pain; Bladder pain syndrome; Histamine glycosaminoglycan; Eosinophil; Comestibles; IC Diet

\section{To the Editor}

Described first around 1808 and documented in 1836 as tic delaroux (painful, idiopathic) disorder of the bladder [1]

Interstitial Cystitis (I.C.) remains a debilitating affliction to the patient and an enigma to the physician.

Interstitial Cystitis, aka, Bladder Pain Syndrome (IC/BPS) affects an estimated 5 to 10 million Americans with a 9 to 1 ratio of women to men [2].

IC/BPS presents as a painful pelvic condition with one or more associated symptoms of urinary: urgency, frequency, dysuria, accompanied on occasion by prostatic pain in men and dyspareunia in women.

The frequency of urination can exceed 60 times a day, causing obvious quality of life issues involving family, work, sleep, travel, driving and sexual activity. Because of embarrassment, patients tend to suffer in silence with a high percentage of patients, 12 percent, considering suicide [3]. The average age of patients with IC/BPS is in the mid-forties with the length of time from the onset of symptoms to correct diagnosis being on average 4 years [4]. The majority of patients with IC/BPS have had long-term misdiagnoses, frequently being treated for nonexistent urinary tract infections. Their gynecological pain has led to unnecessary hysterectomies, as well as other abdominal/gynecological surgeries, which unfortunately, tend to increase IC/BPS intensity.

Patients with IC/BPS frequently deal with several associated disorders including Irritable Bowel Syndrome (IBS), gluten intolerance, fibromyalgia, chronic fatigue, endometriosis, vulvodynia, allergic disorders (including asthma and atopic dermatitis), Sjorgen's syndrome, rheumatoid arthritis, lupus erythematous, chronic sterile prostatitis and anxiety-stress disorders [5].

As of yet no universally accepted cause has been identified. Several loosely associated sets of symptoms have generated theoretical pathogenic implications to mast cells, autoimmune reactions, infection inflammation sequela, atopy and general posttraumatic insults to the body.

Clinical and laboratory evidence does confer compelling support for the role mast cells, and perhaps, eosinophils, may play in the development of symptoms. This concept is hinged on the fact that identifiable histamine, eosinophilic factor and leukotriene release from mast cells in the bladder wall can cause the symptoms of pain, swelling, scarring and impaired mucosal healing seen in IC/BPS patients. Damage to the membrane coating of the bladder, the glycosaminoglycan $(\mathrm{GaG})$ layer, is thought to be a possible result of these inflammatory cell actions [6]. This bladder mucosal damage can allow irritating urinary chemicals to leak into the surrounding tissues causing chronic pain and cyclical inflammation. In response to this pathology, bladder walls of IC/BPS patients tend to have a proliferation of nerve fibers, which accelerate and intensify pain perception. A unique close overlap of pelvic floor innervations results in pain originating in one area (i.e. bladder and urinary tract) to be referred to adjacent organs of the genitourinary system, greatly compounding diagnostic and treatment endeavors [5].

In historical and current observations, attention to causative agents has frequently implicated comestibles (food and drink). These observations propose that the abnormal bladder is abnormally susceptible to irritants in foods, such as spices, potassium, caffeine, and acids. In up to $90 \%$ of IC/BPS patients comestibles have been shown to cause a flare in symptoms [6]. Citrus fruits, tomatoes, vitamin C, artificial sweeteners, caffeine, alcohol, carbonated beverages and spicy foods are frequently implicated [4]. Most physicians who treat IC/BPS patients give those patients an empirical list including these foods and others, to aid in an elimination challenge trial to see which foods from the "IC diet" list cause issues for that particular patient.

The theory of an atopic influence in IC/BPS is alluring. Eosinophilic Cystitis has been identified and with our newer understanding of Eosinophilic Esophagitis and its relationship with atopy a common cause could be carefully postulated. Based on observations made by both clinicians and patients, several strong indications of an atopic influence have been illustrated. These recognitions include the following:

1. Classical allergy symptoms and flares in IC/BPS seem to occur in parallel in a subset of patients.

*Corresponding author: C Steven Smith MD/MS FAAAAI, Director, AAFIC Allersmith Labs Research, 312 South Lyndon Lane, Louisville, Kentucky 40222 , USA, 502-609-2852, Fax: 502-895-3356; E-mail: aaacsmith@gmail.com

Received February 16, 2013; Accepted March 15, 2013; Published March 21 2013

Citation: C Steven Smith, HH Reed (2013) A Relationship of Interstitial Cystitis and Atopy. J Aller Ther 4: 131. doi:10.4172/2155-6121.1000131

Copyright: (C) 2013 C Steven Smith, et al. This is an open-access article distributed under the terms of the Creative Commons Attribution License, which permits unrestricted use, distribution, and reproduction in any medium, provided the original author and source are credited. 
2. Allergy modifying mediators including antihistamines, leukotriene response modifiers, a Th2 cytokine inhibitor (Suplatast tosilate) which down regulates $\operatorname{IgE}$ production and eosinophil inflammation responses and an anti-IgE antibody (omalizumab) have been proven successful in reducing IC/BPS symptoms [7].

3. Intravesical provocation tests with skin prick test and RAST positive food allergens causes an increase in bladder wall histamine release, eosinophil and mast cell counts, and flares of symptoms in many IC/BPS patients, especially those with a concomitant history of asthma [8].

\section{Personal Observations}

With no allergy literature giving reference to atopic causes of urinary tract disorders, I was skeptical when a local urologist and his nurse practitioners, who specialize in IC/BPS, asked me to consider evaluating their patients for an allergic influence to the illness. I agreed to do a few screenings on their patients, expecting to give them the bad news that I could not help with this condition.

The screening involved:

1. A detailed history with special emphasis on foods or pollen seasons which the patient had associated with an increase in their bladder symptoms.

2. Documentation of more classical allergic symptoms and their relationship to the patient's IC/BPS.

3. Documentation of bladder biopsy microscopic studies with regards to stains for Mast cells and Eosinophils.

4. Skin prick test with standard food antigens (Greer Labs) representing foods the patient ate at least 4 times a year. Responses to wheal and flare being measured and recorded on a 1 to 4 scale based on millimeters in diameter.

5. Gluten serology and genetics when the patient's personal or family history indicated a reasonable risk of intolerance or celiac disease.

6. 4-week elimination of foods found positive on Skin prick test followed by singular challenges to each food based on a reintroduction of suspect foods every 7 to 10 days for each food. Those foods, which caused a definite flare in the IC/BPS, were immediately removed from the diet and a recovery period allowed recovering from the flare before proceeding to the next food to be challenged.

7. Patients with plausible associations of their symptoms also underwent environmental allergy testing followed by either environmental control for causative agents or subcutaneous immunotherapy injections.

The first patient I evaluated was a mid-30's white female with a 2 year duration of IC/BPS and a 2 year recurrence of asthma, which had previously been in remission. Skin prick test to several foods produced a rather strong response to corn, a food the patient was not fond of and rarely ingested. The patient shared with me in her history being employed in the office of a local popcorn factory for 2 years and about her daily ingestion of popcorn, which she did like. I took the patient totally off corn products, including popcorn, and within a few weeks, her IC/BPS had greatly improved, along with her asthma. Seeking even better asthma control, I asked the patient to take a 2-week leave from the factory. This resulted in a significant increase in her pulmonary function test value. I was chagrined, and now after seeing over 400 patients for local nurse practitioners, urologists, gynecologists, and primary care physicians, I am convinced there is an atopic factor contributing to IC/BPS in some patients.

For patients with symptoms of IC/BPS, I do a skin prick test screen to all the available foods the patient eats.Since I have learned from experience, several causative foods are not top ten food allergens. I sometimes wonder if our listing of "top" allergy causing foods is a selffulfilling prediction because the vast majority of allergists only test for those foods, leaving many foods allergens without investigation. In certain history indicative cases, I test inhalants as well. Remarkably, several patients who had noted increases in their symptoms of IC/ BPS with seasonal pollenosis have seen parallel improvement in both conditions following an immunotherapy program. A recent analysis of our follow-up records from a cohort of 76 patients, indicate that over $80 \%$ report symptom improvement with addition of allergy care to their armamentarium of treatments.

Success in improving the lives of so many IC/BPS patients has prompted me to this appeal to allergists and immunologists, who possess an expertise to share, to consider evaluating patients suffering with this abstruse, painful, and psychosocially debilitating syndrome.

Certainly a well-designed multi-center, multiple specialties, prospective study is needed to advance and solidify our knowledge. In the interim, we should be open to evaluation of these patients for allergic contributions to their illness.

\section{References}

1. Parsons JK, Parsons CL (2004) The historical origins of interstitial cystitis. J Urol 171: 20-22.

2. Berry SH, Elliott MN, Suttorp M, Bogart LM, Stoto MA, et al. (2011) Prevalence of Symptoms of Bladder Pain Syndrome/Interstitial Cystitis among Adult Females in the United States. J Urol 186: 540-544.

3. Hepner KA, Watkins KE, Elliott MN, Clemens JQ, Hilton LG, et al. (2012) Suicidal Ideation among Patients with Bladder Pain Syndrome/Interstitial Cystitis. Urolology 80: 280-285

4. A.D.A.M/ Medical Encyclopedia (2012) Interstitial Cystitis Pub Med Health

5. Lorenzo Gómez MF, Gómez Castro S (2004) Physiopathologic relationship between interstitial cystitis and rheumatic, autoimmune, and chronic inflammatory diseases. Arch Esp Urol 57: 25-34

6. Friedlander JI, Shorter B, Moldwin RM (2012) Diet and its role in interstitial cystitis/bladder pain syndrome (IC/BPS) and comorbid conditions. BJU Int 109: 1584-1591.

7. A Report of the Bladder Research Progress Review Group (2002) Over Coming Bladder Disease-A Strategic Plan for Research. NIDDKD; NIH.

8. Lee J, Doggweiler-Wiygul R, Kim S, Hill BD, Yoo TJ (2006) Is interstitial cystitis an allergic disorder?: A case of interstitial cystitis treated successfully with antiIgE. Int J Urol 13: 631-634. 